CRYSTALLOGRAPHIC COMMUNICATIONS

ISSN 2056-9890

Received 10 October 2015

Accepted 29 October 2015

Edited by J. Simpson, University of Otago, New Zealand

Keywords: arylboronic acid; hydrogen-bonding interactions; halogen-bonding interactions; biphenyl; THF solvate; crystal structure

CCDC reference: 1434205

Supporting information: this article has supporting information at journals.iucr.org/e

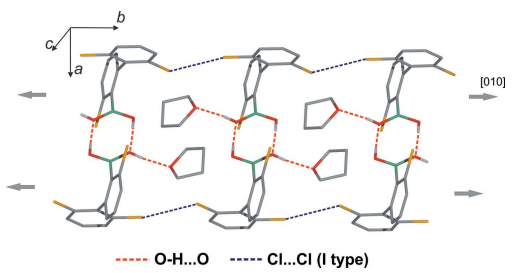

\section{Crystal structure of $\left(2^{\prime}, 3,6^{\prime}\right.$-trichlorobiphenyl-2-yl)- boronic acid tetrahydrofuran monosolvate}

\author{
Krzysztof Durka, ${ }^{*}$ Tomasz Kliś and Janusz Serwatowski
}

Physical Chemistry Department, Faculty of Chemistry, Warsaw University of Technology, Noakowskiego 3, 00-664 Warsaw, Poland. *Correspondence e-mail: kdurka@ch.pw.edu.pl

The title compound, $\mathrm{C}_{12} \mathrm{H}_{8} \mathrm{BCl}_{3} \mathrm{O}_{2} \cdot \mathrm{C}_{4} \mathrm{H}_{8} \mathrm{O}$, crystallizes as a tetrahydrofuran monosolvate. The boronic acid group adopts a syn-anti conformation and is significantly twisted along the carbon-boron bond by $69.2(1)^{\circ}$, due to considerable steric hindrance from the $2^{\prime}, 6^{\prime}$-dichlorophenyl group that is located ortho to the boronic acid substituent. The phenyl rings of the biphenyl are almost perpendicular to one another, with a dihedral angle of $87.9(1)^{\circ}$ between them. In the crystal, adjacent molecules are linked via $\mathrm{O}-\mathrm{H} \cdots \mathrm{O}$ interactions to form centrosymmetric dimers with $R_{2}^{2}(8)$ motifs, which have recently been shown to be energetically very favourable. The hydroxy groups are in an anti conformation and are also engaged in hydrogen-bonding interactions with the $\mathrm{O}$ atom of the tetrahydrofuran solvent molecule. $\mathrm{Cl} \cdots \mathrm{Cl}$ halogen-bonding interactions $[\mathrm{Cl} \cdots \mathrm{Cl}=3.464$ (1) $\AA$ ] link neigbouring dimers into chains running along [010]. Further aggregation occurs due to an additional $\mathrm{Cl} \cdots \mathrm{Cl}$ halogen bond $[\mathrm{Cl} \cdots \mathrm{Cl}=3.387$ (1) $\AA$ ].

\section{Chemical context}

Boronic acids and their derivatives have been studied intensively in recent years due to their numerous applications in organic, analytical and materials chemistry (Hall, 2011; Furukawa \& Yaghi, 2009). They are widely used in medicine, for example, as antifungal and antibacterical agents (Adamczyk-Woźniak et al., 2015; Kane et al., 2003; Vogt et al., 2013). Besides these applications, phenylboronic acids have also been studied in terms of crystal engineering (Nishiyabu et al., 2011; Severin, 2009). In contrast, biphenyl-based boronic acids have been largely neglected. Exceptions to this include reports of the crystal structures of (2-biphenylyl)boronic acid (Filthaus et al., 2008) and (2-methoxy-3-biphenyl)boronic acid (Davies et al., 2008). In this manuscript we focus our attention on a sterically hindered boronic acid derivative based on a biphenyl core with a boronic group located at the 2-position of one benzene ring with a $\mathrm{Cl}$ substituent at the 3-position. The second benzene ring of the biphenyl ring system carries chlorine substituents at the 2- and 6-positions. This molecule crystallized as a 1:1 solvate with THF, Fig. 1.
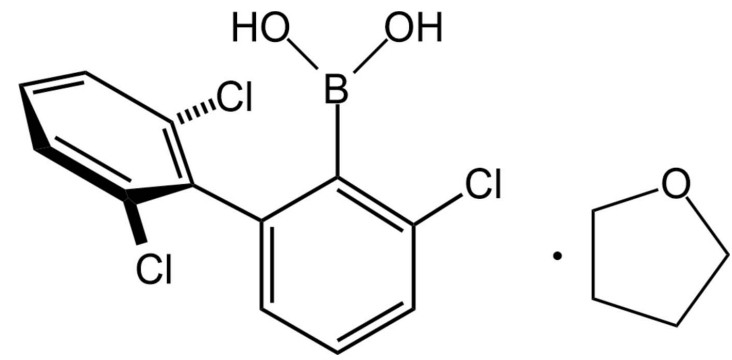


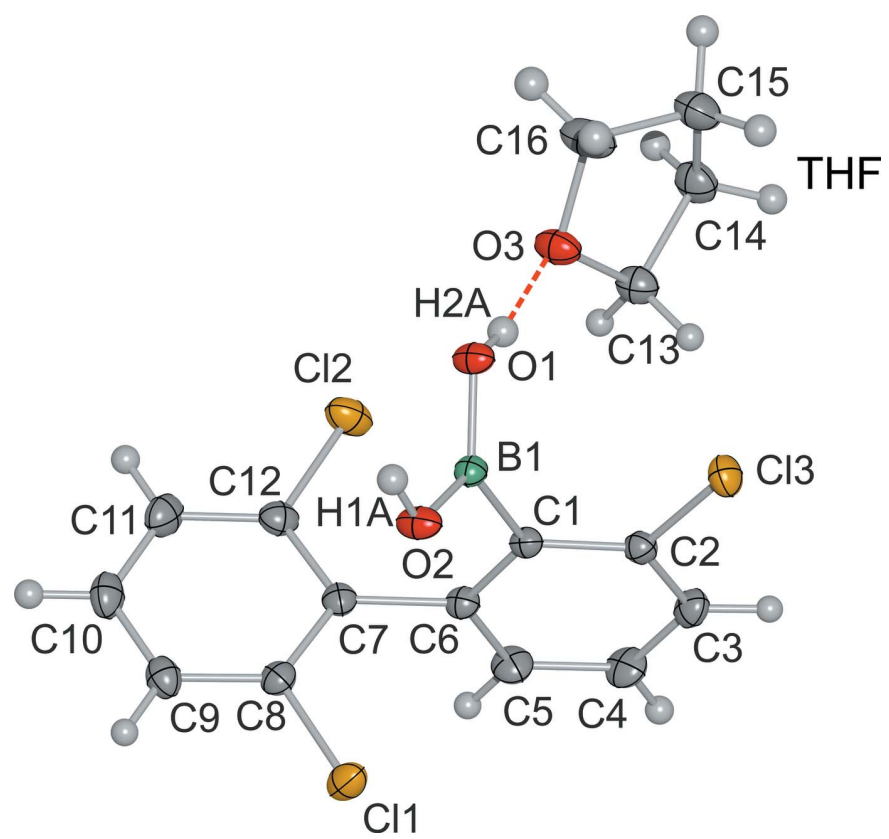

Figure 1

The structure of $\mathbf{1}$, showing the atom numbering, with displacement ellipsoids drawn at the $50 \%$ probability level.

\section{Structural commentary}

The B-C $[1.5907(16) \AA]$ and $\mathrm{B}-\mathrm{O} \quad[1.3514(14)$, 1.3641 (14) $\mathrm{A}$ ] bonds in the title compound (I) are within the expected range typically observed for boronic acids (Madura et al., 2014; Luliński et al., 2007; Maly et al., 2006; Shimpi et al., 2007; Durka et al., 2012). The molecular structure shows that the $\mathrm{B}(\mathrm{OH})_{2}$ group adopts the usual syn-anti conformation (Fig. 1). The boronic acid substituent is significantly rotated about the $\mathrm{C}-\mathrm{B}$ bond in order to minimize the steric hindrance between the boronic group and the adjacent $2^{\prime}, 6^{\prime}$-dichlorophenyl ring $\left[\tau_{\mathrm{C} 2-\mathrm{C} 1-\mathrm{B} 1-\mathrm{O} 1}=69.2(2)^{\circ}\right]$. In the structure of the
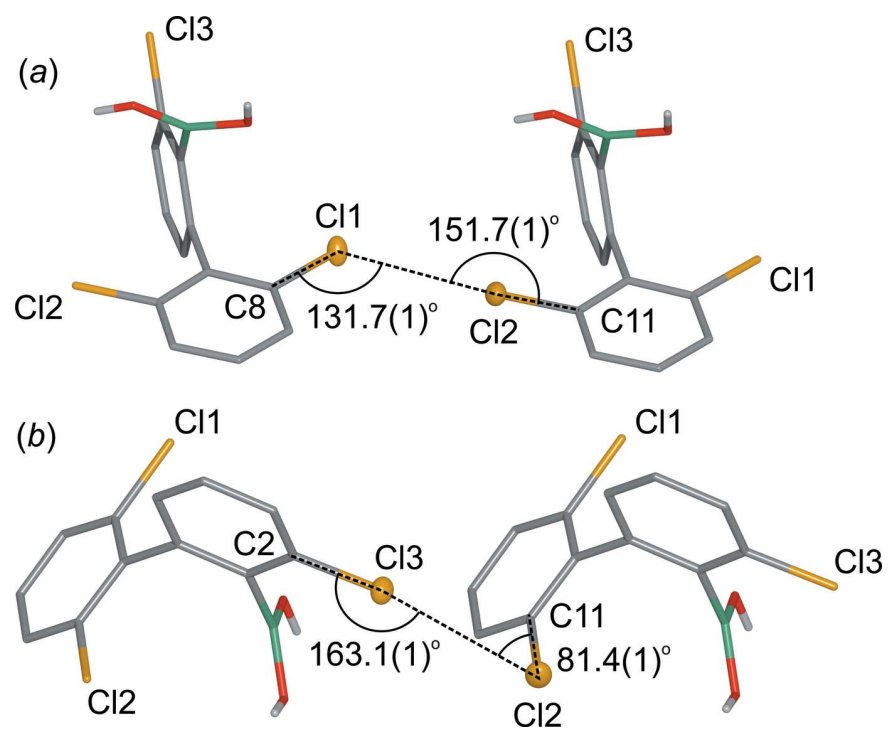

Figure 2

Type I (a) and II $(b) \mathrm{Cl} \cdots \mathrm{Cl}$ halogen bonds in (I).

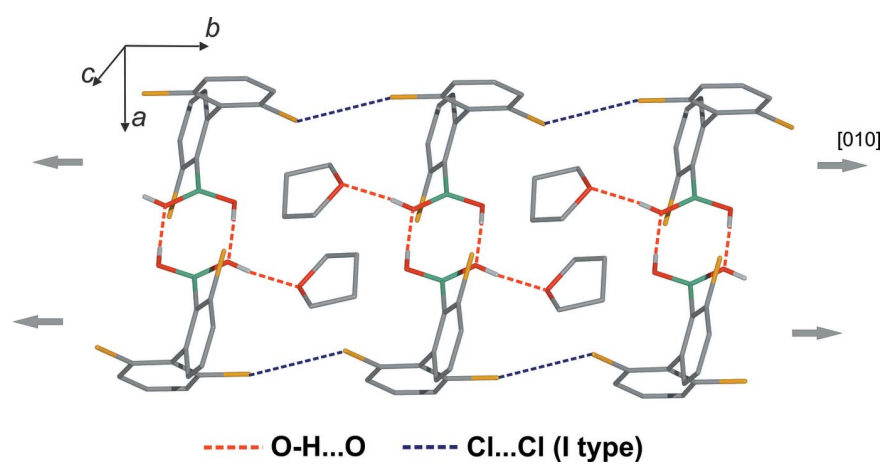

Figure 3

Molecular chains formed along $b$ by type $1 \mathrm{Cl} \cdots \mathrm{Cl}$ halogen bonds. Also shown are inversion dimers and inclusion of the solvent through $\mathrm{O}-$ $\mathrm{H} \cdots \mathrm{O}$ hydrogen bonds. Aromatic $\mathrm{H}$ atoms have been omitted for clarity.

related (2-biphenylyl)boronic acid (Filthaus et al., 2008) this torsion angle is some $20^{\circ}$ smaller, which clearly shows the influence of the three chlorine substituents on this structure. It is also notable that in (I) the phenyl rings of the biphenyl system are almost perpendicular to one another $\left[\tau_{\mathrm{C} 1-\mathrm{C} 6-\mathrm{C} 7-}\right.$ $\mathrm{C} 11=87.9(1)^{\circ}$ ], whereas in (2-biphenylyl)boronic acid they are rotated by only 48.4 or $45.4^{\circ}$ for the two unique molecules in the asymmetric unit.

\section{Supramolecular features}

In the crystal, centrosymmetric $\mathrm{O}-\mathrm{H} \cdots \mathrm{O}$ hydrogen-bonded dimers are formed. The anti-oriented $\mathrm{OH}$ group is engaged in an intermolecular $\mathrm{O}-\mathrm{H} \cdots \mathrm{O}$ hydrogen bond (Table 1) with the oxygen atom from the tetrahydrofuran solvate molecule. Because all of the hydrogen-bond acceptor centres are saturated, the syn $\mathrm{OH}$ group is not involved in any side hydrogenbond interactions. Neighbouring dimers are connected through $\mathrm{Cl} \cdots \mathrm{Cl}$ halogen bonds $\left[d_{\mathrm{Cl} \cdots \mathrm{Cl}}=3.464\right.$ (1) $\AA$; the sum of the van der Waals radii for $\mathrm{Cl}$ is $3.50 \AA]$. In terms of geometry of this contact, it can be classified as a type I halogen bond (Fig. 2a), (Metrangolo et al., 2005; Nayak et al., 2011). These contacts result in the formation of molecular chains propagating along [010] (Fig. 3). A three-dimensional network forms through additional $\mathrm{Cl} \cdots \mathrm{Cl}$ halogen bonds (Fig. 4) of type II $\left[d_{\mathrm{Cl} \ldots \mathrm{Cl}}=3.387(1) \AA\right]$ (Fig. $\left.2 b\right)$.

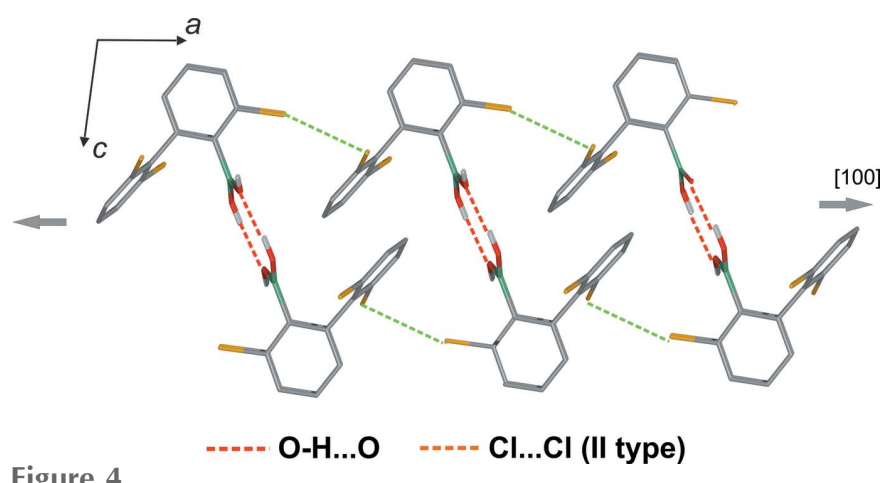

Figure 4

Crystal packing showing intermolecular $\mathrm{O}-\mathrm{H} \cdots \mathrm{O}$ and type $\mathrm{II} \mathrm{Cl} \cdots \mathrm{Cl}$ interactions. 
Table 1

Hydrogen-bond geometry $\left(\AA,^{\circ}\right)$.

\begin{tabular}{lllll}
\hline$D-\mathrm{H} \cdots A$ & $D-\mathrm{H}$ & $\mathrm{H} \cdots A$ & $D \cdots A$ & $D-\mathrm{H} \cdots A$ \\
\hline $\mathrm{O} 1-\mathrm{H} 1 A \cdots \mathrm{O} 3$ & $0.82(1)$ & $1.84(1)$ & $2.6475(12)$ & $169(2)$ \\
$\mathrm{O} 2-\mathrm{H} 2 A \cdots \mathrm{O} 1^{\mathrm{i}}$ & $0.84(1)$ & $1.96(1)$ & $2.7997(12)$ & $175(2)$ \\
\hline
\end{tabular}

Symmetry code: (i) $-x+2,-y+1,-z+1$.

\section{Synthesis and crystallization}

Synthesis of (I) (Fig. 5): A solution of 2-iodo-2',3,6'-trichlorobiphenyl $(3.8 \mathrm{~g}, 10 \mathrm{mmol})$ in THF $(50 \mathrm{~mL})$ was added to a stirred solution of $n$-BuLi $(10 \mathrm{mmol})$ in THF $(30 \mathrm{~mL})$ at $195 \mathrm{~K}$. The resulting colorless solution was stirred for $1 \mathrm{~h}$ to give a colorless precipitate. The electrophile, $\mathrm{B}(\mathrm{OMe})_{3}(2.1 \mathrm{~g}$, $20 \mathrm{mmol}$ ) was then added to the stirred mixture to give a colorless solution which was stirred for $1 \mathrm{~h}$ and then hydrolyzed with $\mathrm{H}_{2} \mathrm{O}(100 \mathrm{~mL})$. Dilute aq. $\mathrm{H}_{2} \mathrm{SO}_{4}$ was added until the $\mathrm{pH}$ was slightly acidic. $\mathrm{Et}_{2} \mathrm{O}(50 \mathrm{~mL})$ was next added and the mixture stirred for $10 \mathrm{~min}$. The organic phase was separated and the aqueous phase was extracted with $\mathrm{Et}_{2} \mathrm{O}(20 \mathrm{~mL})$. The combined organic solutions were dried over $\mathrm{MgSO}_{4}$ and evaporated to give a colorless precipitate, yield $2.0 \mathrm{~g}(66 \%)$. ${ }^{1} \mathrm{H}$ NMR (400 MHz, acetone- $\left.d_{6}\right): \delta=8.00(2 \mathrm{H}, s, \mathrm{OH}), 7.51$ $(2 \mathrm{H}, m), 7.39(3 \mathrm{H}, m), 7.05(1 \mathrm{H}, \mathrm{m}) ;{ }^{13} \mathrm{C}\{1 \mathrm{H}\} \quad \mathrm{NMR}$ (100.6 MHz, acetone- $\left.d_{6}\right): \delta=141.49,139.62,139$ (br), 136.17, $134.84,130.49,129.99,128.24,128.14,127.53$.

Crystals suitable for X-ray diffraction analysis were grown by slow evaporation of a THF solution.

\section{Refinement details}

Crystal data, data collection and structure refinement details are summarized in Table 2. All $\mathrm{CH}$ hydrogen atoms were placed in calculated positions with $\mathrm{C}-\mathrm{H}$ distances of 0.95 or $0.99 \AA$. They were included in the refinement in the ridingmotion approximation with $U_{\text {iso }}($ phenyl $\mathrm{H})=1.2 U_{\text {eq }}(\mathrm{C})$. The positions of the $\mathrm{OH}$ hydrogen atoms were first found in a difference map. Then their bond lengths were restrained in the last least-squares cycles, with an $\mathrm{O}-\mathrm{H}$ distance of $0.85 \AA$ and their coordinates refined with $U_{\text {iso }}($ hydroxyl $\mathrm{H})=1.5 U_{\text {eq }}(\mathrm{O})$.

\section{Acknowledgements}

The X-ray measurements were undertaken in the Crystallographic Unit of the Physical Chemistry Laboratory at the Chemistry Department of the University of Warsaw. This

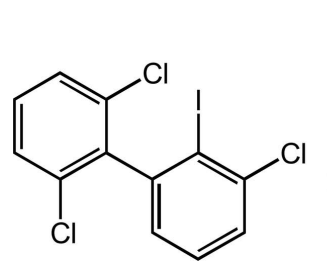

$$
\begin{aligned}
& \text { 1. n-BuLi, THF, }-78{ }^{\circ} \mathrm{C} \\
& \text { 2. } \mathrm{B}(\mathrm{OMe})_{3},-78{ }^{\circ} \mathrm{C} \\
& \mathrm{MeOH}, \mathrm{Li}_{2} \mathrm{SO}_{4}, \mathrm{BuI}
\end{aligned}
$$

\begin{tabular}{|c|c|}
\hline \multicolumn{2}{|l|}{ Crystal data } \\
\hline Chemical formula & $\mathrm{C}_{12} \mathrm{H}_{8} \mathrm{BCl}_{3} \mathrm{O}_{2} \cdot \mathrm{C}_{4} \mathrm{H}_{8} \mathrm{O}$ \\
\hline$M_{\mathrm{r}}$ & 373.45 \\
\hline Crystal system, space group & Triclinic, $P \overline{1}$ \\
\hline Temperature $(\mathrm{K})$ & 130 \\
\hline$a, b, c(\AA)$ & $8.3306(3), 8.7122(2), 12.4307$ (4) \\
\hline$\alpha, \beta, \gamma\left({ }^{\circ}\right)$ & $98.683(3), 97.737(3), 99.398(3)$ \\
\hline$V\left(\AA^{3}\right)$ & $868.07(5)$ \\
\hline$Z$ & 2 \\
\hline Radiation type & Mo $K \alpha$ \\
\hline$\mu\left(\mathrm{mm}^{-1}\right)$ & 0.54 \\
\hline Crystal size (mm) & $0.15 \times 0.12 \times 0.10$ \\
\hline \multicolumn{2}{|l|}{ Data collection } \\
\hline Diffractometer & $\begin{array}{l}\text { Agilent SuperNova Dual Source } \\
\text { diffractometer with an Atlas } \\
\text { detector }\end{array}$ \\
\hline Absorption correction & $\begin{array}{l}\text { Multi-scan (CrysAlis PRO; } \\
\text { Agilent, 2014) }\end{array}$ \\
\hline$T_{\min }, T_{\max }$ & $0.795,1.000$ \\
\hline $\begin{array}{l}\text { No. of measured, independent and } \\
\text { observed }[I>2 \sigma(I)] \text { reflections }\end{array}$ & $28647,6107,5162$ \\
\hline$R_{\text {int }}$ & 0.032 \\
\hline$(\sin \theta / \lambda)_{\max }\left(\AA^{-1}\right)$ & 0.758 \\
\hline \multicolumn{2}{|l|}{ Refinement } \\
\hline$R\left[F^{2}>2 \sigma\left(F^{2}\right)\right], w R\left(F^{2}\right), S$ & $0.034,0.085,1.05$ \\
\hline No. of reflections & 6107 \\
\hline No. of parameters & 214 \\
\hline No. of restraints & 2 \\
\hline $\mathrm{H}$-atom treatment & $\begin{array}{l}\mathrm{H} \text { atoms treated by a mixture of } \\
\text { independent and constrained } \\
\text { refinement }\end{array}$ \\
\hline$\Delta \rho_{\max }, \Delta \rho_{\min }\left(\mathrm{e} \AA^{-3}\right)$ & $0.45,-0.36$ \\
\hline
\end{tabular}

Figure 5

The synthesis of 2-chloro-6-(2',6'-dichlorophenyl)phenylboronic acid.
Table 2

Experimental details.

Computer programs: CrysAlis PRO (Agilent, 2014), SHELXS97 (Sheldrick, 2015), SHELXL2013 (Sheldrick, 2015), DIAMOND (Brandenburg, 2005) and publCIF (Westrip, 2010).

work was supported by the Aldrich Chemical Company through the donation of chemicals and equipment, and by the Warsaw University of Technology.

\section{References}

Adamczyk-Woźniak, A., Borys, K. M. \& Sporzyński, A. (2015). Chem. Rev. 115, 5224-5247.

Agilent (2014). CrysAlis PRO. Agilent Technologies, Santa Clara, USA.

Brandenburg, K. (2005). DIAMOND. Crystal Impact GbR, Bonn, Germany.

Davies, C. J., Gregory, A., Griffith, P., Perkins, T., Singh, K. \& Solan, G. A. (2008). Tetrahedron, 64, 9857-9864.

Durka, K., Jarzembska, K. N., Kamiński, R., Luliński, S., Serwatowski, J. \& Woźniak, K. (2012). Cryst. Growth Des. 12, 3720-3734.

Filthaus, M., Oppel, I. M. \& Bettinger, H. F. (2008). Org. Biomol. Chem. 6, 1201-1207.

Furukawa, H. \& Yaghi, O. M. (2009). J. Am. Chem. Soc. 131, 88758883.

Hall, D. G. (2011). In Boronic Acids. Weinheim: Wiley-VCH.

Kane, R. C., Bross, P. F., Farrell, A. T. \& Pazdur, R. (2003). Oncologist, 8, 508-513.

Luliński, S., Madura, I., Serwatowski, J., Szatyłowicz, H. \& Zachara, J. (2007). New J. Chem. 31, 144-154.

Madura, I., Czerwińska, K. \& Sołdańska, D. (2014). Cryst. Growth Des. 14, 5912-5921.

Maly, K. E., Maris, T. \& Wuest, J. D. (2006). CrystEngComm, 8, 33-35. 
Metrangolo, P., Neukirch, H., Pilati, T. \& Resnati, G. (2005). Acc. Chem. Res. 38, 386-395.

Nayak, S. K., Reddy, M. K., Guru Row, T. N. \& Chopra, D. (2011). Cryst. Growth Des. 11, 1578-1596.

Nishiyabu, R., Kubo, Y., James, T. D. \& Fossey, J. S. (2011). Chem. Commun. 47, 1124-1150.

Severin, K. (2009). Dalton Trans. pp. 5254-5264.
Sheldrick, G. M. (2015). Acta Cryst. C71, 3-8.

Shimpi, M. R., SeethaLekshmi, N. \& Pedireddi, V. R. (2007). Cryst. Growth Des. 7, 1958-1963.

Vogt, F. G., Williams, G. R. \& Copley, R. C. B. (2013). J. Pharm. Sci. 102, 3705-3716.

Westrip, S. P. (2010). J. Appl. Cryst. 43, 920-925. 


\section{supporting information}

Acta Cryst. (2015). E71, 1471-1474 [https://doi.org/10.1107/S205698901502054X]

\section{Crystal structure of $\left(2^{\prime}, 3,6^{\prime}\right.$-trichlorobiphenyl-2-yl) boronic acid tetrahydrofuran monosolvate}

\section{Krzysztof Durka, Tomasz Kliś and Janusz Serwatowski}

\section{Computing details}

Data collection: CrysAlis PRO (Agilent, 2014); cell refinement: CrysAlis PRO (Agilent, 2014); data reduction: CrysAlis PRO (Agilent, 2014); program(s) used to solve structure: SHELXS97 (Sheldrick, 2015); program(s) used to refine structure: SHELXL2013 (Sheldrick, 2015); molecular graphics: DIAMOND (Brandenburg, 2005); software used to prepare material for publication: publCIF (Westrip, 2010).

(2',3,6'-Trichlorobiphenyl-2-yl)boronic acid tetrahydrofuran monosolvate

Crystal data

$\mathrm{C}_{12} \mathrm{H}_{8} \mathrm{BCl}_{3} \mathrm{O}_{2} \cdot \mathrm{C}_{4} \mathrm{H}_{8} \mathrm{O}$

$M_{r}=373.45$

Triclinic, $P \overline{1}$

$a=8.3306$ (3) $\AA$

$b=8.7122(2) \AA$

$c=12.4307$ (4) $\AA$

$\alpha=98.683(3)^{\circ}$

$\beta=97.737(3)^{\circ}$

$\gamma=99.398(3)^{\circ}$

$V=868.07(5) \AA^{3}$

$Z=2$

\section{Data collection}

Agilent SuperNova Dual Source diffractometer with an Atlas detector

Radiation source: SuperNova (Mo) X-ray Source

Mirror monochromator

Detector resolution: 5.2195 pixels $\mathrm{mm}^{-1}$

$\omega$ scans

Absorption correction: multi-scan

(CrysAlis PRO; Agilent, 2014)

\section{Refinement}

Refinement on $F^{2}$

Least-squares matrix: full

$R\left[F^{2}>2 \sigma\left(F^{2}\right)\right]=0.034$

$w R\left(F^{2}\right)=0.085$

$S=1.05$

6107 reflections

214 parameters

2 restraints
$F(000)=384$

$D_{\mathrm{x}}=1.429 \mathrm{Mg} \mathrm{m}^{-3}$

Melting point: $411 \mathrm{~K}$

Mo $K \alpha$ radiation, $\lambda=0.71073 \AA$

Cell parameters from 14113 reflections

$\theta=2.4-32.4^{\circ}$

$\mu=0.54 \mathrm{~mm}^{-1}$

$T=130 \mathrm{~K}$

Fragment, colourless

$0.15 \times 0.12 \times 0.10 \mathrm{~mm}$

$T_{\min }=0.795, T_{\max }=1.000$

28647 measured reflections

6107 independent reflections

5162 reflections with $I>2 \sigma(I)$

$R_{\text {int }}=0.032$

$\theta_{\max }=32.6^{\circ}, \theta_{\min }=2.4^{\circ}$

$h=-12 \rightarrow 12$

$k=-13 \rightarrow 13$

$l=-18 \rightarrow 18$

Hydrogen site location: mixed

$\mathrm{H}$ atoms treated by a mixture of independent and constrained refinement

$w=1 /\left[\sigma^{2}\left(F_{\mathrm{o}}^{2}\right)+(0.0328 P)^{2}+0.3549 P\right]$

where $P=\left(F_{\mathrm{o}}^{2}+2 F_{\mathrm{c}}^{2}\right) / 3$

$(\Delta / \sigma)_{\max }=0.001$

$\Delta \rho_{\max }=0.45$ e $\AA^{-3}$

$\Delta \rho_{\min }=-0.36 \mathrm{e} \AA^{-3}$ 


\section{Special details}

Experimental. Absorption correction: CrysAlisPro (Agilent Technologies, 2014), Empirical absorption correction using spherical harmonics, implemented in SCALE3 ABSPACK scaling algorithm.

Geometry. All e.s.d.'s (except the e.s.d. in the dihedral angle between two 1.s. planes) are estimated using the full covariance matrix. The cell e.s.d.'s are taken into account individually in the estimation of e.s.d.'s in distances, angles and torsion angles; correlations between e.s.d.'s in cell parameters are only used when they are defined by crystal symmetry. An approximate (isotropic) treatment of cell e.s.d.'s is used for estimating e.s.d.'s involving 1.s. planes.

Fractional atomic coordinates and isotropic or equivalent isotropic displacement parameters $\left(\AA^{2}\right)$

\begin{tabular}{|c|c|c|c|c|}
\hline & $x$ & $y$ & $z$ & $U_{\text {iso }} * / U_{\text {eq }}$ \\
\hline $\mathrm{C} 1$ & $0.77416(13)$ & $0.33187(12)$ & $0.22248(8)$ & $0.01544(18)$ \\
\hline $\mathrm{C} 2$ & $0.85513(13)$ & $0.33777(12)$ & $0.13113(9)$ & $0.01761(19)$ \\
\hline $\mathrm{C} 3$ & $0.77605(15)$ & $0.28436(14)$ & $0.02290(9)$ & $0.0221(2)$ \\
\hline $\mathrm{H} 3$ & 0.8355 & 0.2914 & -0.0368 & $0.027^{*}$ \\
\hline $\mathrm{C} 5$ & $0.52304(15)$ & $0.21255(15)$ & $0.09167(10)$ & 0.0233 \\
\hline H5 & 0.4085 & 0.1690 & 0.0782 & $0.028 *$ \\
\hline C6 & $0.60414(13)$ & $0.26808(13)$ & $0.19972(9)$ & $0.01703(19)$ \\
\hline $\mathrm{C} 7$ & $0.50529(13)$ & $0.26201(13)$ & $0.29144(9)$ & $0.01742(19)$ \\
\hline $\mathrm{C} 8$ & $0.47656(14)$ & $0.12861(13)$ & $0.34104(9)$ & $0.0188(2)$ \\
\hline C9 & $0.38144(14)$ & $0.12012(15)$ & $0.42447(10)$ & $0.0230(2)$ \\
\hline H9 & 0.3646 & 0.0276 & 0.4565 & $0.028^{*}$ \\
\hline $\mathrm{C} 10$ & $0.31167(15)$ & $0.24852(16)$ & $0.46023(11)$ & $0.0263(2)$ \\
\hline H10 & 0.2466 & 0.2441 & 0.5172 & $0.032 *$ \\
\hline C11 & $0.43238(14)$ & $0.38827(13)$ & $0.33025(10)$ & $0.0211(2)$ \\
\hline $\mathrm{C} 12$ & $0.33610(15)$ & $0.38351(16)$ & $0.41341(11)$ & $0.0262(2)$ \\
\hline H12 & 0.2879 & 0.4714 & 0.4377 & $0.031 *$ \\
\hline $\mathrm{C} 13$ & $0.60897(16)$ & $0.22065(15)$ & $0.00366(10)$ & $0.0257(2)$ \\
\hline H13 & 0.5531 & 0.1825 & -0.0697 & $0.031^{*}$ \\
\hline C14 & $0.82695(16)$ & $0.75012(14)$ & $0.14313(10)$ & $0.0241(2)$ \\
\hline H14A & 0.7067 & 0.7154 & 0.1184 & $0.029^{*}$ \\
\hline H14B & 0.8843 & 0.6702 & 0.1077 & $0.029^{*}$ \\
\hline $\mathrm{C} 15$ & $0.88574(17)$ & $0.91105(15)$ & $0.11452(11)$ & 0.0283 \\
\hline H15A & 0.8014 & 0.9785 & 0.1202 & $0.034^{*}$ \\
\hline H15B & 0.9142 & 0.9012 & 0.0393 & $0.034^{*}$ \\
\hline $\mathrm{C} 16$ & $1.03885(18)$ & $0.97668(16)$ & $0.20241(12)$ & 0.0325 \\
\hline H16A & 1.1369 & 0.9378 & 0.1804 & $0.039^{*}$ \\
\hline H16B & 1.0621 & 1.0937 & 0.2165 & $0.039^{*}$ \\
\hline $\mathrm{C} 17$ & $0.9899(2)$ & $0.91251(17)$ & $0.30259(12)$ & 0.0358 \\
\hline H17A & 1.0868 & 0.8873 & 0.3471 & $0.043^{*}$ \\
\hline H17B & 0.9443 & 0.9914 & 0.3495 & $0.043^{*}$ \\
\hline B1 & $0.86796(14)$ & $0.39672(14)$ & $0.34516(10)$ & $0.0160(2)$ \\
\hline $\mathrm{O} 1$ & 0.92407 (11) & $0.55444(9)$ & $0.38238(7)$ & $0.02050(16)$ \\
\hline $\mathrm{O} 2$ & 0.89019 (11) & $0.29285(10)$ & $0.41379(7)$ & 0.02293 \\
\hline $\mathrm{O} 3$ & $0.86646(12)$ & $0.77103(10)$ & $0.26144(7)$ & $0.02631(19)$ \\
\hline Cl1 & $0.56394(4)$ & $-0.03377(3)$ & $0.29838(2)$ & $0.02648(7)$ \\
\hline $\mathrm{Cl} 2$ & 0.46307 (4) & $0.55927(3)$ & $0.27352(3)$ & $0.02936(8)$ \\
\hline $\mathrm{Cl} 3$ & $1.06696(3)$ & $0.41387(4)$ & $0.15296(2)$ & $0.02402(7)$ \\
\hline
\end{tabular}




\begin{tabular}{lllll} 
H1A & $0.902(2)$ & $0.6121(19)$ & $0.3383(13)$ & $0.036^{*}$ \\
H2A & $0.941(2)$ & $0.336(2)$ & $0.4767(12)$ & $0.036^{*}$ \\
\hline
\end{tabular}

Atomic displacement parameters $\left(\AA^{2}\right)$

\begin{tabular}{lllllll}
\hline & $U^{11}$ & $U^{22}$ & $U^{33}$ & $U^{12}$ & $U^{13}$ & $U^{23}$ \\
\hline C1 & $0.0179(5)$ & $0.0142(4)$ & $0.0137(4)$ & $0.0028(3)$ & $0.0005(3)$ & $0.0028(3)$ \\
C2 & $0.0196(5)$ & $0.0163(4)$ & $0.0168(5)$ & $0.0029(4)$ & $0.0027(4)$ & $0.0034(4)$ \\
C3 & $0.0289(6)$ & $0.0237(5)$ & $0.0148(5)$ & $0.0061(4)$ & $0.0047(4)$ & $0.0040(4)$ \\
C5 & $0.0212(5)$ & $0.0264(6)$ & $0.0181(5)$ & $0.0002(4)$ & $-0.0030(4)$ & $0.0013(4)$ \\
C6 & $0.0186(5)$ & $0.0169(4)$ & $0.0146(4)$ & $0.0025(4)$ & $0.0002(4)$ & $0.0028(4)$ \\
C7 & $0.0156(4)$ & $0.0194(5)$ & $0.0152(4)$ & $0.0009(4)$ & $0.0001(3)$ & $0.0015(4)$ \\
C8 & $0.0197(5)$ & $0.0202(5)$ & $0.0150(5)$ & $0.0025(4)$ & $0.0003(4)$ & $0.0014(4)$ \\
C9 & $0.0208(5)$ & $0.0284(6)$ & $0.0192(5)$ & $0.0013(4)$ & $0.0022(4)$ & $0.0065(4)$ \\
C10 & $0.0186(5)$ & $0.0363(7)$ & $0.0236(6)$ & $0.0033(5)$ & $0.0060(4)$ & $0.0044(5)$ \\
C11 & $0.0176(5)$ & $0.0196(5)$ & $0.0243(5)$ & $0.0016(4)$ & $0.0010(4)$ & $0.0027(4)$ \\
C12 & $0.0193(5)$ & $0.0296(6)$ & $0.0293(6)$ & $0.0065(4)$ & $0.0056(4)$ & $0.0005(5)$ \\
C13 & $0.0305(6)$ & $0.0290(6)$ & $0.0138(5)$ & $0.0024(5)$ & $-0.0024(4)$ & $0.0013(4)$ \\
C14 & $0.0267(6)$ & $0.0221(5)$ & $0.0226(5)$ & $0.0031(4)$ & $0.0000(4)$ & $0.0059(4)$ \\
C15 & $0.0332(7)$ & $0.0258(6)$ & $0.0278(6)$ & $0.0044(5)$ & $0.0057(5)$ & $0.0114(5)$ \\
C16 & $0.0333(7)$ & $0.0243(6)$ & $0.0375(7)$ & $-0.0023(5)$ & $0.0049(6)$ & $0.0068(5)$ \\
C17 & $0.0411(8)$ & $0.0261(6)$ & $0.0328(7)$ & $-0.0066(5)$ & $-0.0064(6)$ & $0.0072(5)$ \\
B1 & $0.0152(5)$ & $0.0170(5)$ & $0.0146(5)$ & $0.0022(4)$ & $0.0009(4)$ & $0.0015(4)$ \\
O1 & $0.0278(4)$ & $0.0162(4)$ & $0.0149(4)$ & $0.0019(3)$ & $-0.0030(3)$ & $0.0027(3)$ \\
O2 & $0.0302(4)$ & $0.0179(4)$ & $0.0170(4)$ & $0.0010(3)$ & $-0.0053(3)$ & $0.0036(3)$ \\
O3 & $0.0328(5)$ & $0.0202(4)$ & $0.0233(4)$ & $-0.0005(3)$ & $-0.0017(3)$ & $0.0072(3)$ \\
C11 & $0.04097(17)$ & $0.02093(13)$ & $0.02001(13)$ & $0.00986(11)$ & $0.00736(11)$ & $0.00481(10)$ \\
C12 & $0.02627(15)$ & $0.02037(13)$ & $0.04284(18)$ & $0.00462(10)$ & $0.00663(12)$ & $0.00884(12)$ \\
C13 & $0.01988(13)$ & $0.02816(14)$ & $0.02462(14)$ & $0.00239(10)$ & $0.00640(10)$ & $0.00636(11)$ \\
& & & & & & \\
\hline
\end{tabular}

Geometric parameters $\left(A,{ }^{\circ}\right)$

\begin{tabular}{llll}
\hline $\mathrm{C} 1-\mathrm{C} 2$ & $1.3999(15)$ & $\mathrm{C} 11-\mathrm{Cl}$ & $1.7388(12)$ \\
$\mathrm{C} 1-\mathrm{C} 6$ & $1.4081(15)$ & $\mathrm{C} 12-\mathrm{H} 12$ & 0.9500 \\
$\mathrm{C} 1-\mathrm{B} 1$ & $1.5907(16)$ & $\mathrm{C} 13-\mathrm{H} 13$ & 0.9500 \\
$\mathrm{C} 2-\mathrm{C} 3$ & $1.3902(16)$ & $\mathrm{C} 14-\mathrm{O} 3$ & $1.4400(15)$ \\
$\mathrm{C} 2-\mathrm{C} 3$ & $1.7498(11)$ & $\mathrm{C} 14-\mathrm{C} 15$ & $1.5183(17)$ \\
$\mathrm{C} 3-\mathrm{C} 13$ & $1.3859(18)$ & $\mathrm{C} 14-\mathrm{H} 14 \mathrm{~A}$ & 0.9900 \\
$\mathrm{C} 3-\mathrm{H} 3$ & 0.9500 & $\mathrm{C} 14-\mathrm{H} 14 \mathrm{~B}$ & 0.9900 \\
$\mathrm{C} 5-\mathrm{C} 13$ & $1.3900(17)$ & $\mathrm{C} 15-\mathrm{C} 16$ & $1.532(2)$ \\
$\mathrm{C} 5-\mathrm{C} 6$ & $1.3954(15)$ & $\mathrm{C} 15-\mathrm{H} 15 \mathrm{~A}$ & 0.9900 \\
$\mathrm{C} 5-\mathrm{H} 5$ & 0.9500 & $\mathrm{C} 15-\mathrm{H} 15 \mathrm{~B}$ & 0.9900 \\
$\mathrm{C} 6-\mathrm{C} 7$ & $1.4959(15)$ & $\mathrm{C} 16-\mathrm{C} 17$ & $1.517(2)$ \\
$\mathrm{C} 7-\mathrm{C} 11$ & $1.3959(16)$ & $\mathrm{C} 16-\mathrm{H} 16 \mathrm{~A}$ & 0.9900 \\
$\mathrm{C} 7-\mathrm{C} 8$ & $1.3976(15)$ & $\mathrm{C} 16-\mathrm{H} 16 \mathrm{~B}$ & 0.9900 \\
$\mathrm{C} 8-\mathrm{C} 9$ & $1.3908(16)$ & $\mathrm{C} 17-\mathrm{O} 3$ & $1.4465(16)$ \\
$\mathrm{C} 8-\mathrm{C} 11$ & $1.7395(12)$ & $\mathrm{C} 17-\mathrm{H} 17 \mathrm{~A}$ & 0.9900 \\
$\mathrm{C} 9-\mathrm{C} 10$ & $1.3851(18)$ & $\mathrm{C} 17-\mathrm{H} 17 \mathrm{~B}$ & 0.9900
\end{tabular}




\begin{tabular}{|c|c|c|c|}
\hline C9-H9 & 0.9500 & $\mathrm{~B} 1-\mathrm{O} 2$ & $1.3514(14)$ \\
\hline $\mathrm{C} 10-\mathrm{C} 12$ & $1.3869(19)$ & $\mathrm{B} 1-\mathrm{O} 1$ & $1.3641(14)$ \\
\hline $\mathrm{C} 10-\mathrm{H} 10$ & 0.9500 & $\mathrm{O} 1-\mathrm{H} 1 \mathrm{~A}$ & $0.821(14)$ \\
\hline $\mathrm{C} 11-\mathrm{C} 12$ & $1.3924(17)$ & $\mathrm{O} 2-\mathrm{H} 2 \mathrm{~A}$ & $0.838(14)$ \\
\hline $\mathrm{C} 2-\mathrm{C} 1-\mathrm{C} 6$ & $116.31(9)$ & $\mathrm{C} 3-\mathrm{C} 13-\mathrm{C} 5$ & $120.03(11)$ \\
\hline $\mathrm{C} 2-\mathrm{C} 1-\mathrm{B} 1$ & $121.95(9)$ & $\mathrm{C} 3-\mathrm{C} 13-\mathrm{H} 13$ & 120.0 \\
\hline $\mathrm{C} 6-\mathrm{C} 1-\mathrm{B} 1$ & $121.73(9)$ & $\mathrm{C} 5-\mathrm{C} 13-\mathrm{H} 13$ & 120.0 \\
\hline $\mathrm{C} 3-\mathrm{C} 2-\mathrm{C} 1$ & $123.29(10)$ & $\mathrm{O} 3-\mathrm{C} 14-\mathrm{C} 15$ & $105.36(10)$ \\
\hline $\mathrm{C} 3-\mathrm{C} 2-\mathrm{Cl} 3$ & $117.77(9)$ & $\mathrm{O} 3-\mathrm{C} 14-\mathrm{H} 14 \mathrm{~A}$ & 110.7 \\
\hline $\mathrm{C} 1-\mathrm{C} 2-\mathrm{C} 13$ & $118.93(8)$ & $\mathrm{C} 15-\mathrm{C} 14-\mathrm{H} 14 \mathrm{~A}$ & 110.7 \\
\hline $\mathrm{C} 13-\mathrm{C} 3-\mathrm{C} 2$ & $118.82(11)$ & $\mathrm{O} 3-\mathrm{C} 14-\mathrm{H} 14 \mathrm{~B}$ & 110.7 \\
\hline $\mathrm{C} 13-\mathrm{C} 3-\mathrm{H} 3$ & 120.6 & $\mathrm{C} 15-\mathrm{C} 14-\mathrm{H} 14 \mathrm{~B}$ & 110.7 \\
\hline $\mathrm{C} 2-\mathrm{C} 3-\mathrm{H} 3$ & 120.6 & $\mathrm{H} 14 \mathrm{~A}-\mathrm{C} 14-\mathrm{H} 14 \mathrm{~B}$ & 108.8 \\
\hline $\mathrm{C} 13-\mathrm{C} 5-\mathrm{C} 6$ & $120.36(11)$ & $\mathrm{C} 14-\mathrm{C} 15-\mathrm{C} 16$ & $102.13(10)$ \\
\hline $\mathrm{C} 13-\mathrm{C} 5-\mathrm{H} 5$ & 119.8 & $\mathrm{C} 14-\mathrm{C} 15-\mathrm{H} 15 \mathrm{~A}$ & 111.3 \\
\hline $\mathrm{C} 6-\mathrm{C} 5-\mathrm{H} 5$ & 119.8 & $\mathrm{C} 16-\mathrm{C} 15-\mathrm{H} 15 \mathrm{~A}$ & 111.3 \\
\hline $\mathrm{C} 5-\mathrm{C} 6-\mathrm{C} 1$ & $121.18(10)$ & $\mathrm{C} 14-\mathrm{C} 15-\mathrm{H} 15 \mathrm{~B}$ & 111.3 \\
\hline $\mathrm{C} 5-\mathrm{C} 6-\mathrm{C} 7$ & $118.41(10)$ & $\mathrm{C} 16-\mathrm{C} 15-\mathrm{H} 15 \mathrm{~B}$ & 111.3 \\
\hline $\mathrm{C} 1-\mathrm{C} 6-\mathrm{C} 7$ & $120.40(9)$ & $\mathrm{H} 15 \mathrm{~A}-\mathrm{C} 15-\mathrm{H} 15 \mathrm{~B}$ & 109.2 \\
\hline $\mathrm{C} 11-\mathrm{C} 7-\mathrm{C} 8$ & $116.15(10)$ & $\mathrm{C} 17-\mathrm{C} 16-\mathrm{C} 15$ & $102.58(11)$ \\
\hline $\mathrm{C} 11-\mathrm{C} 7-\mathrm{C} 6$ & $121.66(10)$ & $\mathrm{C} 17-\mathrm{C} 16-\mathrm{H} 16 \mathrm{~A}$ & 111.3 \\
\hline $\mathrm{C} 8-\mathrm{C} 7-\mathrm{C} 6$ & $122.17(10)$ & $\mathrm{C} 15-\mathrm{C} 16-\mathrm{H} 16 \mathrm{~A}$ & 111.3 \\
\hline $\mathrm{C} 9-\mathrm{C} 8-\mathrm{C} 7$ & $122.68(11)$ & $\mathrm{C} 17-\mathrm{C} 16-\mathrm{H} 16 \mathrm{~B}$ & 111.3 \\
\hline $\mathrm{C} 9-\mathrm{C} 8-\mathrm{Cl} 1$ & $118.09(9)$ & $\mathrm{C} 15-\mathrm{C} 16-\mathrm{H} 16 \mathrm{~B}$ & 111.3 \\
\hline $\mathrm{C} 7-\mathrm{C} 8-\mathrm{Cl1}$ & $119.23(9)$ & $\mathrm{H} 16 \mathrm{~A}-\mathrm{C} 16-\mathrm{H} 16 \mathrm{~B}$ & 109.2 \\
\hline $\mathrm{C} 10-\mathrm{C} 9-\mathrm{C} 8$ & $119.03(11)$ & $\mathrm{O} 3-\mathrm{C} 17-\mathrm{C} 16$ & $106.63(11)$ \\
\hline $\mathrm{C} 10-\mathrm{C} 9-\mathrm{H} 9$ & 120.5 & $\mathrm{O} 3-\mathrm{C} 17-\mathrm{H} 17 \mathrm{~A}$ & 110.4 \\
\hline $\mathrm{C} 8-\mathrm{C} 9-\mathrm{H} 9$ & 120.5 & $\mathrm{C} 16-\mathrm{C} 17-\mathrm{H} 17 \mathrm{~A}$ & 110.4 \\
\hline $\mathrm{C} 9-\mathrm{C} 10-\mathrm{C} 12$ & $120.49(11)$ & $\mathrm{O} 3-\mathrm{C} 17-\mathrm{H} 17 \mathrm{~B}$ & 110.4 \\
\hline $\mathrm{C} 9-\mathrm{C} 10-\mathrm{H} 10$ & 119.8 & $\mathrm{C} 16-\mathrm{C} 17-\mathrm{H} 17 \mathrm{~B}$ & 110.4 \\
\hline $\mathrm{C} 12-\mathrm{C} 10-\mathrm{H} 10$ & 119.8 & $\mathrm{H} 17 \mathrm{~A}-\mathrm{C} 17-\mathrm{H} 17 \mathrm{~B}$ & 108.6 \\
\hline $\mathrm{C} 12-\mathrm{C} 11-\mathrm{C} 7$ & $122.63(11)$ & $\mathrm{O} 2-\mathrm{B} 1-\mathrm{O} 1$ & $119.71(10)$ \\
\hline $\mathrm{C} 12-\mathrm{C} 11-\mathrm{Cl} 2$ & $118.30(9)$ & $\mathrm{O} 2-\mathrm{B} 1-\mathrm{C} 1$ & $118.96(9)$ \\
\hline $\mathrm{C} 7-\mathrm{C} 11-\mathrm{C} 12$ & $119.07(9)$ & $\mathrm{O} 1-\mathrm{B} 1-\mathrm{C} 1$ & $121.33(9)$ \\
\hline $\mathrm{C} 10-\mathrm{C} 12-\mathrm{C} 11$ & $119.01(12)$ & $\mathrm{B} 1-\mathrm{O} 1-\mathrm{H} 1 \mathrm{~A}$ & $115.3(13)$ \\
\hline $\mathrm{C} 10-\mathrm{C} 12-\mathrm{H} 12$ & 120.5 & $\mathrm{~B} 1-\mathrm{O} 2-\mathrm{H} 2 \mathrm{~A}$ & $113.1(12)$ \\
\hline $\mathrm{C} 11-\mathrm{C} 12-\mathrm{H} 12$ & 120.5 & $\mathrm{C} 14-\mathrm{O} 3-\mathrm{C} 17$ & $109.77(10)$ \\
\hline $\mathrm{C} 6-\mathrm{C} 1-\mathrm{C} 2-\mathrm{C} 3$ & $-0.15(16)$ & $\mathrm{C} 11-\mathrm{C} 8-\mathrm{C} 9-\mathrm{C} 10$ & $179.55(9)$ \\
\hline $\mathrm{B} 1-\mathrm{C} 1-\mathrm{C} 2-\mathrm{C} 3$ & $-178.97(10)$ & $\mathrm{C} 8-\mathrm{C} 9-\mathrm{C} 10-\mathrm{C} 12$ & $0.09(18)$ \\
\hline $\mathrm{C} 6-\mathrm{C} 1-\mathrm{C} 2-\mathrm{Cl} 3$ & $-179.42(8)$ & $\mathrm{C} 8-\mathrm{C} 7-\mathrm{C} 11-\mathrm{C} 12$ & $-0.07(17)$ \\
\hline $\mathrm{B} 1-\mathrm{C} 1-\mathrm{C} 2-\mathrm{C} 13$ & $1.76(14)$ & $\mathrm{C} 6-\mathrm{C} 7-\mathrm{C} 11-\mathrm{C} 12$ & $178.17(11)$ \\
\hline $\mathrm{C} 1-\mathrm{C} 2-\mathrm{C} 3-\mathrm{C} 13$ & $-0.56(17)$ & $\mathrm{C} 8-\mathrm{C} 7-\mathrm{C} 11-\mathrm{Cl} 2$ & $179.60(8)$ \\
\hline $\mathrm{Cl} 3-\mathrm{C} 2-\mathrm{C} 3-\mathrm{C} 13$ & $178.72(9)$ & $\mathrm{C} 6-\mathrm{C} 7-\mathrm{C} 11-\mathrm{Cl} 2$ & $-2.15(15)$ \\
\hline $\mathrm{C} 13-\mathrm{C} 5-\mathrm{C} 6-\mathrm{C} 1$ & $-0.63(18)$ & $\mathrm{C} 9-\mathrm{C} 10-\mathrm{C} 12-\mathrm{C} 11$ & $-0.26(19)$ \\
\hline $\mathrm{C} 13-\mathrm{C} 5-\mathrm{C} 6-\mathrm{C} 7$ & $177.91(11)$ & $\mathrm{C} 7-\mathrm{C} 11-\mathrm{C} 12-\mathrm{C} 10$ & $0.25(18)$ \\
\hline $\mathrm{C} 2-\mathrm{C} 1-\mathrm{C} 6-\mathrm{C} 5$ & $0.74(16)$ & $\mathrm{C} 12-\mathrm{C} 11-\mathrm{C} 12-\mathrm{C} 10$ & $-179.42(10)$ \\
\hline
\end{tabular}




$\begin{array}{llll}\mathrm{B} 1-\mathrm{C} 1-\mathrm{C} 6-\mathrm{C} 5 & 179.56(10) & \mathrm{C} 2-\mathrm{C} 3-\mathrm{C} 13-\mathrm{C} 5 & 0.69(18) \\ \mathrm{C} 2-\mathrm{C} 1-\mathrm{C} 6-\mathrm{C} 7 & -177.77(9) & \mathrm{C} 6-\mathrm{C} 5-\mathrm{C} 13-\mathrm{C} 3 & -0.11(19) \\ \mathrm{B} 1-\mathrm{C} 1-\mathrm{C} 6-\mathrm{C} 7 & 1.05(15) & \mathrm{O} 3-\mathrm{C} 14-\mathrm{C} 15-\mathrm{C} 16 & 33.84(13) \\ \mathrm{C} 5-\mathrm{C} 6-\mathrm{C} 7-\mathrm{C} 11 & -90.69(13) & \mathrm{C} 14-\mathrm{C} 15-\mathrm{C} 16-\mathrm{C} 17 & -35.53(14) \\ \mathrm{C} 1-\mathrm{C} 6-\mathrm{C} 7-\mathrm{C} 11 & 87.86(13) & \mathrm{C} 15-\mathrm{C} 16-\mathrm{C} 17-\mathrm{O} 3 & 25.23(15) \\ \mathrm{C} 5-\mathrm{C} 6-\mathrm{C} 7-\mathrm{C} 8 & 87.44(14) & \mathrm{C} 2-\mathrm{C} 1-\mathrm{B} 1-\mathrm{O} 2 & -111.66(12) \\ \mathrm{C} 1-\mathrm{C} 6-\mathrm{C} 7-\mathrm{C} 8 & -94.00(13) & \mathrm{C} 6-\mathrm{C} 1-\mathrm{B} 1-\mathrm{O} 2 & 69.59(14) \\ \mathrm{C} 11-\mathrm{C} 7-\mathrm{C} 8-\mathrm{C} 9 & -0.11(16) & \mathrm{C} 2-\mathrm{C} 1-\mathrm{B} 1-\mathrm{O} 1 & 69.17(14) \\ \mathrm{C} 6-\mathrm{C} 7-\mathrm{C} 8-\mathrm{C} 9 & -178.35(10) & \mathrm{C} 6-\mathrm{C} 1-\mathrm{B} 1-\mathrm{O} 1 & -109.58(12) \\ \mathrm{C} 11-\mathrm{C} 7-\mathrm{C} 8-\mathrm{C} 11 & -179.56(8) & \mathrm{C} 15-\mathrm{C} 14-\mathrm{O} 3-\mathrm{C} 17 & -18.86(14) \\ \mathrm{C} 6-\mathrm{C} 7-\mathrm{C} 8-\mathrm{C} 11 & 2.21(14) & \mathrm{C} 16-\mathrm{C} 17-\mathrm{O} 3-\mathrm{C} 14 & -4.30(16) \\ \mathrm{C} 7-\mathrm{C} 8-\mathrm{C} 9-\mathrm{C} 10 & 0.10(17) & & \end{array}$

Hydrogen-bond geometry $\left(\AA,{ }^{\circ}\right)$

\begin{tabular}{lllll}
\hline$D-\mathrm{H} \cdots A$ & $D-\mathrm{H}$ & $\mathrm{H} \cdots A$ & $D \cdots A$ & $D-\mathrm{H} \cdots A$ \\
\hline $\mathrm{O} 1-\mathrm{H} 1 A \cdots \mathrm{O} 3$ & $0.82(1)$ & $1.84(1)$ & $2.6475(12)$ & $169(2)$ \\
$\mathrm{O} 2-\mathrm{H} 2 A \cdots \mathrm{O} 1^{\mathrm{i}}$ & $0.84(1)$ & $1.96(1)$ & $2.7997(12)$ & $175(2)$ \\
\hline
\end{tabular}

Symmetry code: (i) $-x+2,-y+1,-z+1$. 